\title{
Data Fusion Strategy for Precise Vehicle Location for Intelligent Self-Aware Maintenance Systems
}

\author{
M. Bevilacqua \\ Cranfield University, \\ Cranfield, Bedfordshire, MK430AL, \\ United Kingdom \\ m.bevilacqua@cranfield.ac.uk
}

\author{
A. Tsourdos, A. Starr, I. Durazo-Cardenas \\ Cranfield University, \\ Cranfield, Bedfordshire, MK430AL, \\ United Kingdom \\ $\{$ a.tsourdos, a.starr, \\ i.s.durazocardenas\}@cranfield.ac.uk
}

\begin{abstract}
Nowadays careful measurement applications are handed over to Wired and Wireless Sensor Network. Taking the scenario of train location as an example, this would lead to an increase in uncertainty about position related to sensors with long acquisition times like Balises, RFID and Transponders along the track. We take into account the data without any synchronization protocols, for increase the accuracy and reduce the uncertainty after the data fusion algorithms. The case studies, we have analysed, derived from the needs of the project partners: train localization, head of an auger in the drilling sector localization and the location of containers of radioactive material waste in a reprocessing nuclear plant. They have the necessity to plan the maintenance operations of their infrastructure basing through architecture that taking input from the sensors, which are localization and diagnosis, maps and cost, to optimize the cost effectiveness and reduce the time of operation.
\end{abstract}

Keywords-data, fusion, strathegy, rail, network, maintenance, location, uncertainty.

\section{INTRODUCTION}

Nowadays careful measurement applications are handed over to Wired and Wireless Sensor Network. In most of these tasks sensor nodes work together to recognize the data fusion process. Synchronization is a critical element in this scenario. Nodes have to be regulated to a common clock and regulated among them. Many synchronization algorithms can be found in literature and some of them have been intentionally established for low cost structural designing where efficient memory management and reduced computational burden are important constraints.

However, the synchronization protocols slow down the elaboration process in order to align the timing of data supply at the source with the slowest sampling time. Taking the scenario of train location as an example, this would lead to an increase in uncertainty about position related to sensors with long acquisition times like Balises, RFID and Transponders along the track.

For this reason we take into account the data without any synchronization protocols, precisely for increase the accuracy and reduce the uncertainty after the data fusion algorithms we will implement and develop.

The data fusion of unsynchronized data sources achieve the goal we have set for the localization scenarios, this, however, involves a more complex management of the resources and requires a clear and precise definition of a strategy for data fusion related to the type of environment and sensors involved in every different scenario.

We can give a definition of unsynchronized sensor data: "Data provided by a sensor of which the output is without any correlation with other sensors in the same net, with different sampling time, period and phase."

\section{LITERATURE REVIEW}

Correct decision making (taking) in the security sector mainly depends on information, received from multiple sources. Often, the information is insufficient, unreliable and contradictive.

Sensor fusion is the combining of sensory data such that the resulting information is in some sense better than would be possible when these sources were used individually, better means: more accurate, more complete, or more dependable.

The first and the most important remark is that fusion process is necessary most of all to reduce (to filter) input information through its integration (merging) and generalization.

Fusion process is necessary to improve accuracy and reduce uncertainty [1].

A number of authors [2-5] have comprehensively reviewed data fusion models and architectures

In the following table some different data fusion strategies are shown: 
TABLE I. DATA FUSION MODELS

\begin{tabular}{|c|c|c|}
\hline Data Fusion Model & Advantages & Disadvantages \\
\hline $\begin{array}{l}\text { JDL data fusion model } \\
\qquad[6,7,8]\end{array}$ & $\begin{array}{l}\text { The sources provide } \\
\text { information ranging } \\
\text { from sensor data to a } \\
\text { priori information from } \\
\text { databases to human } \\
\text { input. } \\
\text { Source pre-processing } \\
\text { enables fusion process to } \\
\text { concentrate on the data } \\
\text { most pertinent, reducing } \\
\text { the processing load. } \\
\text { The database } \\
\text { management system's } \\
\text { task is to monitor, } \\
\text { evaluate, add, update } \\
\text { and provide information } \\
\text { for the fusion processes. } \\
\text { Human-computer } \\
\text { Interaction provides an } \\
\text { interface for human } \\
\text { input and } \\
\text { communication. }\end{array}$ & $\begin{array}{l}\text { - It does not address } \\
\text { multi-image fusion } \\
\text { problems } \\
\text { - Sensors involving } \\
\text { multiple components } \\
\text { not supported }\end{array}$ \\
\hline $\begin{array}{c}\text { Dasarathy's functional } \\
\text { model } \\
{[9,10]}\end{array}$ & $\begin{array}{l}\text { Levels of abstraction } \\
\text { - Data } \\
\text { - Feature } \\
\text { - Decision } \\
\text { - Categorization of data } \\
\text { fusion functions in terms } \\
\text { of the type of data level } \\
\text { at input/output. }\end{array}$ & $\begin{array}{l}\text { No particular } \\
\text { problems }\end{array}$ \\
\hline $\begin{array}{c}\text { Waterfall fusion process } \\
\text { model } \\
{[11,12]}\end{array}$ & $\begin{array}{c}\text { - Fusion process in } \\
\text { stages } \\
\text { - Omission of feedback } \\
\text { data flow is the major } \\
\text { limitation }\end{array}$ & $\begin{array}{l}\text { No particular } \\
\text { problems }\end{array}$ \\
\hline $\begin{array}{c}\text { Boyd Loop } \\
\text { [13,14] }\end{array}$ & $\begin{array}{c}\text { - OODA cycle } \\
\text { Comparative OODA - } \\
\text { JDL: } \\
\text { Observe: source pre- } \\
\text { processing } \\
\text { Orientate: levels } 1 \text { to } 3 \\
\text { Decide: level } 4\end{array}$ & $\begin{array}{c}\text { Act: no direct } \\
\text { counterpart in the JDL } \\
\text { model }\end{array}$ \\
\hline $\begin{array}{c}\text { Thomopoulos' Fusion } \\
\text { Model } \\
{[15]}\end{array}$ & $\begin{array}{l}\text { An architecture based on } \\
\text { three data processing } \\
\text { levels: the signal level, } \\
\text { the level of evidence and } \\
\text { the level of dynamics. }\end{array}$ & $\begin{array}{l}\text { Mathematical model } \\
\text { that describes the } \\
\text { process from which } \\
\text { data is collected must } \\
\text { be known }\end{array}$ \\
\hline $\begin{array}{c}\text { Durrant-Whyte } \\
\text { architecture } \\
{[16]}\end{array}$ & $\begin{array}{l}\text { An architecture oriented } \\
\text { towards robot systems. } \\
\text { Common Representation } \\
\text { Format } \\
\text { The data from all the } \\
\text { sensors is converted to } \\
\text { this CRF and fused by a } \\
\text { high-level fusion model }\end{array}$ & $\begin{array}{l}\text { Each sensor must } \\
\text { perform its own } \\
\text { conversion, what } \\
\text { makes necessary a } \\
\text { sensor model. }\end{array}$ \\
\hline
\end{tabular}

\begin{tabular}{|c|c|c|}
\hline $\begin{array}{c}\text { The "Omnibus" process } \\
\text { model }\end{array}$ & $\begin{array}{c}\text { Model defines the } \\
\text { ordering of processes } \\
\text { and makes the cycle } \\
\text { explicit } \\
\text { [17] }\end{array}$ & $\begin{array}{c}\text { No particular } \\
\text { problems }\end{array}$ \\
\hline $\begin{array}{c}\text { Provides a much more } \\
\text { fine-grained structuring } \\
\text { of the processing levels } \\
\text { than the Boyd loop. }\end{array}$ \\
\hline $\begin{array}{c}\text { Endsley's Situation } \\
\text { Awareness }\end{array}$ & $\begin{array}{c}\text { The model that has two } \\
\text { main parts: } \\
\text { - The situation } \\
\text { awareness core } \\
\text { - Various sets of factors } \\
\text { affecting the core. }\end{array}$ & \\
\hline 18,19$]$ & $\begin{array}{c}\text { No particular } \\
\text { problems }\end{array}$ \\
\hline
\end{tabular}

In the following table some mathematical solution commonly used for the data fusion are compared in terms od advantages and disadvantages [1]:

\section{TABLE II. MATHEMATICAL SOLUTION FOR DATA FUSION}

Mathematical
Solution for Data
Fusion

Bayesian Network

[20-24]

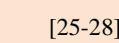

[25-28]

Information filter

[29-31]
A Bayesian network is a probabilistic graphical model (a type of statistical model) that represents a set of random variables and their conditional dependencies via a directed acyclic graph (DAG).

Incorporate noise effects (both measurement and modelling) Recursive computational structure

Explicit description of process and observations allows different sensor models to be incorporated within the basic $\mathrm{KF}$ algorithm.

Use of statistical measures of uncertainty makes it possible to quantitatively evaluate the role each sensor plays in the overall system performance using error covariance matrix $P$

Compared with Kalman filter, the measurement update of information filter is identical with that of Kalman filter

Can be readily implemented for heterogeneous sensors

Can be a measure to check observability

Can easily cope with the correlation

\section{Conditional} probability distribution for each variable must be known.

The Kalman filter in general is not an optimal estimator if the initial estimate of the state is wrong, or if the process is modeled incorrectly, the filter may quickly diverge, owing to its linearization.

The estimated covariance matrix tends to underestimate the true covariance matrix and therefore risks becoming inconsistent in the statistical sense without the addition of "stabilizing noise".

\section{Closely associated} with the Fisher information measures

Cannot take the correlation into account 


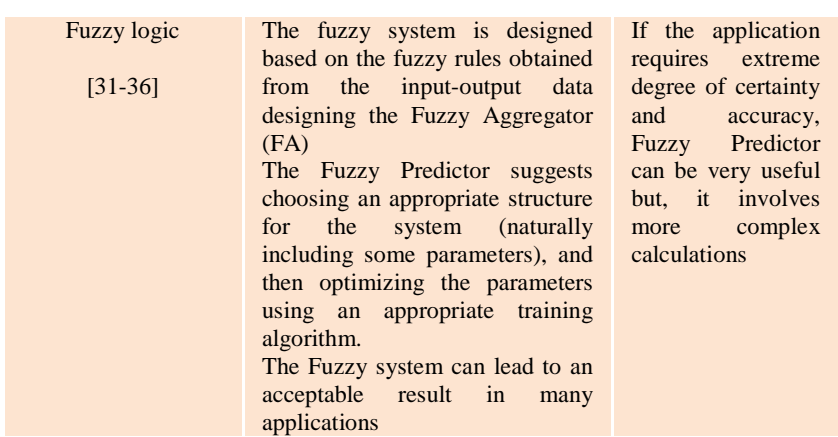

\section{CASE Studies}

The cases study we have analyzed are derived from the needs, in the scenario of localization, of the three main project partners: localization: in railway network, the location of the head of an auger in the drilling sector and the last one is about the location of containers of radioactive material waste in a reprocessing nuclear plant.

The generic scheme of the case studies will later be developed it has been studied in detail and a strategy has been developed for data fusion related to the needs of our industrial partners. All partners have the necessity to be able to plan the maintenance operations of their infrastructure basing through an architecture that taking input from the sensors, which are localization and diagnosis, maps and cost, will enable them to optimize the cost effectiveness and reduce the time of operation. We think we can achieve this aims finding the best data fusion strategy to reduce the uncertainty in location scenario.

In the figure 1 the demo for the whole project are represented and in figure 2 the sensor fusion block are drown more in detail.

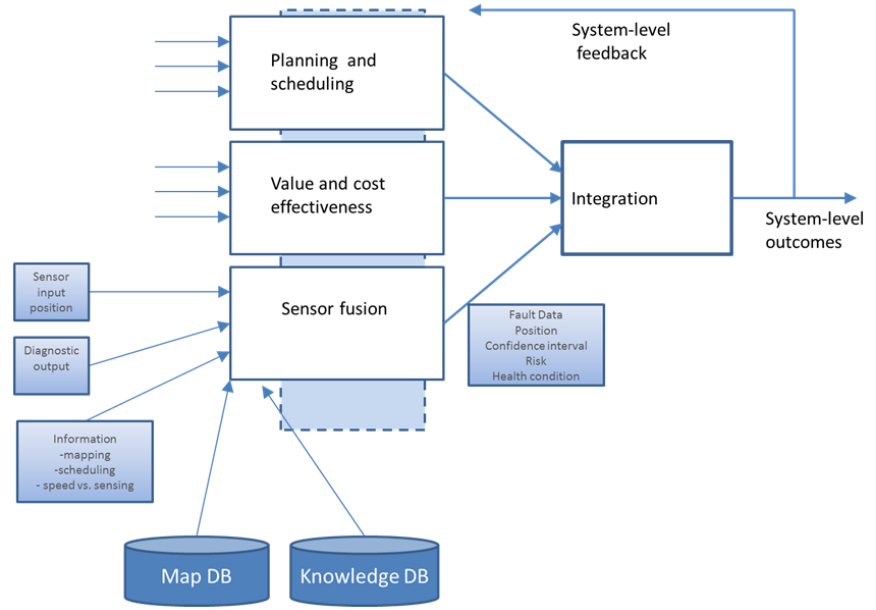

Fig. 1. Demo for the whole project

The particularization of the Sensor fusion block in figure 2 includes inputs like we see in figure 1. Also the Knowledge DB and Map DB must be particularized for each case that we will see in details in the next figures 3,4 and 5 .

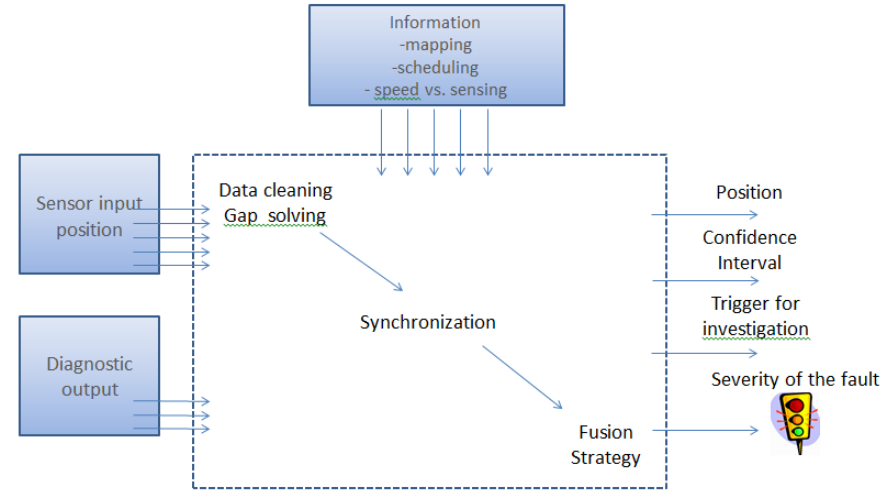

Fig. 2. Generic scheme

\section{THE PROPOSAL}

Now we analyze and suggest a data fusion strategy for one of each scenario we talked in the previous paragraph.

\section{1) Railway Industry}

The positional accuracy target of the UK future rail is $<2 \mathrm{~m}$ [37]. However; a finer resolution is required for locating faults such as damage or missing parts. A critical consideration of these requirements has been the capability to resolve train occupancy in adjacent tracks, with a high degree of confidence.

In response to these needs of the Network Rail Industry, it will be necessary to formalize and implement a demo that allows us by using the correct strategy for data fusion to obtain these objectives. We can achieve this by having as input position signals from IMU, GPS odometer, RFID, Balises and diagnostic signals from laser scanners, ultrasound combined to generate the desired output and at the end plan maintenance.

Taking into account all these parameters and sensors we can decide to use a data fusion strategy like JDL or the Omnibus process model. In figure 3 a particularization of the case is represented.

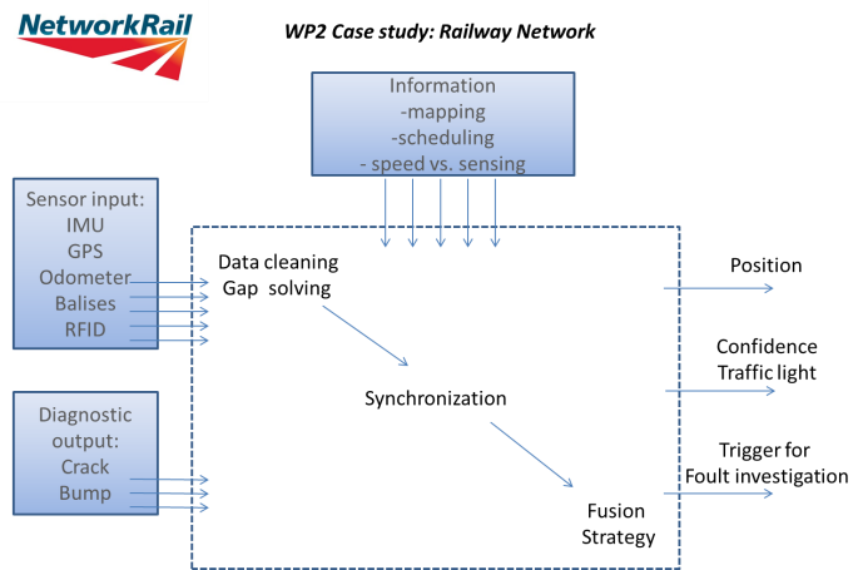

Fig. 3. Network Rail case study 


\section{2) Nuclear Reprocessing Plant}

Also with regard to the maintenance schedule in the operations of reprocessing of fissile material, the choice of the best technique of data fusion can make it more secure and reduce costs, which, starting from the analysis of the monitoring signals of the systems involved can generate the required output in the minimum time.

The main Sellafield challenges that must be overcome can be enumerated here:

- $\quad$ Pond characterization - remote robots to measure, vacuum up sludge

- $\quad$ Tank characterization - hand held monitors.

- Building char - "suck" the radioactive contamination from structures

- Contaminated ground - moles underground to target and extract intermediate level waste to avoid contamination in ground water

- $\quad$ Monitoring drums and packing - stores patrolled by intelligent robots able to monitor, repair, remove failed drums etc.

Taking into account all these parameters and sensors we can decide to use a data fusion strategy like JDL or DurrantWhyte architecture that is really feasible for underwater robots estimation problems. In figure 4 a particularization of the second case is represented.

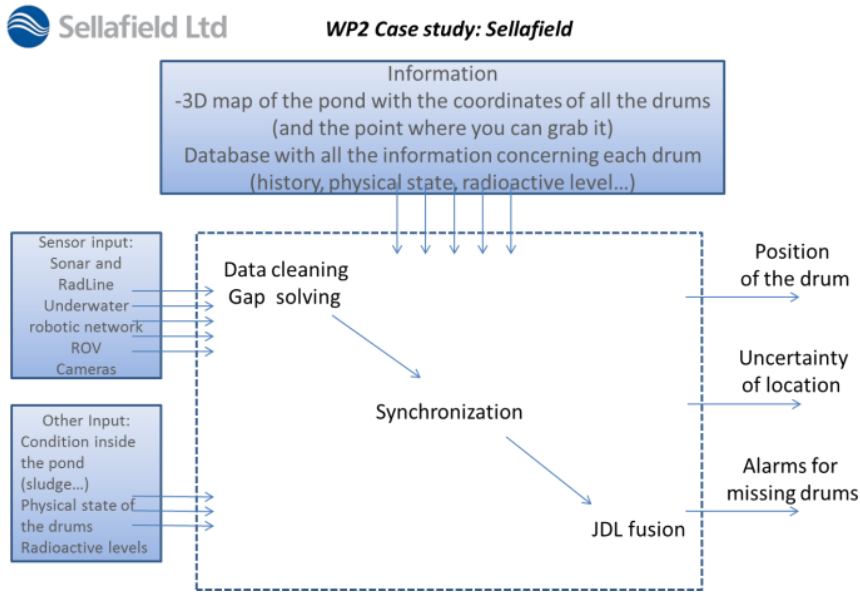

Fig. 4. Nuclear reprocessing case study

\section{3) Drilling}

Schlumberger is one of the leading oil and gas Extraction Company, they have many problems related to the localization of the exact position of the head of their drilling in order to find, in the minimum time and without any retry, the oilfield they who are trying to reach.

The challenge is relate to the harsh environments they work on. Usually this is the typical environments: $500 \mathrm{~g}$, 200C +, no GPS, tight spaces. For deep drilling also $15<20 \mathrm{~km}$, fracking 10,000 wells/year shallow drilling; decommissioning.

They also want to go over the following problems: they want to remove as much people as they can from drilling rigs and give more intelligence to their automatics equipment. They also have really poor observed systems with lots of uncertainty, for this reason they want to find the right balance between planning and uncertainty taking into account also the cost.

Taking into account all these parameters and sensors we can decide to use a data fusion strategy like Waterfall fusion process model or JDL model using Kalman filter that is really good for this problem where we want to achieve a data fusion strategy onboard and real time. In figure 5 a particularization of the last case is represented.

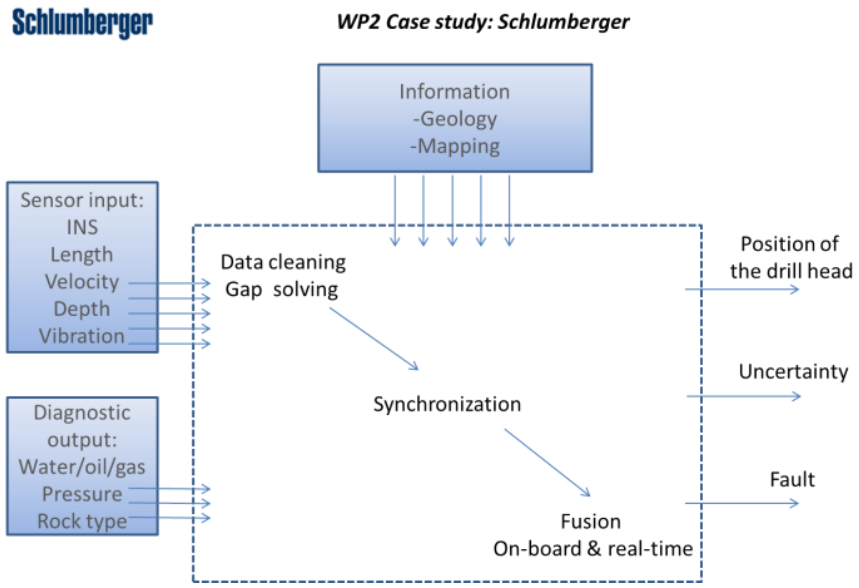

Fig. 5. Drilling case study

\section{CONCLUSION AND FUTURE WORKS}

In conclusion, different data fusion techniques were studied and analyzed. The best data fusion technique was applied to the each of the three case studies based on the characteristics of the input, output and aims that each scenario requires.

The implementation of the selected fusion strategy allows achieving the minimum accuracy in location and guaranteeing the company to plan the maintenance with the minimum impact on their budget.

The next step on this project and this research it will be to apply the different schemes, which we applied before only in simulation with these demos, also with the real data, to understand if we can achieve the same performances also in real time, without interfering with the normal procedures of the partners.

\section{ACKNOWLEDGMENT}

Authors wish to thank two MSc students: Piotr Karol Jurewicz and Guillemette Weymuller for the help given during the phase of scenarios study and the literature review.

\section{REFERENCES}

[1] Precise vehicle location as a fundamental parameter for intelligent self-aware rail-track maintenance systems - Durazo-Cardenas, A Starr, A. Tsourdos, M.Bevilacqua - 3rd International Conference on Through-life Engineering Services 
[2] P. Hui Foo and G. Wah Ng, High level information fusion: An overview, Journal of Advances in Information Fusion, 8 (1), (2013) pp. 33-72.

[3] D.L. Hall and J. Llinas, An introduction to multisensor data fusion, Proceedings of the IEEE, 85 (1), (1997) pp. 6-23.

[4] J. Esteban, A. Starr, R. Willetts, P. Hannah and P. Bryanston-Cross, A review of data fusion models and architechtures: Towards engineering guidelines, Neural Comput \& Applic, 14 (4), (2005) pp. 273-281.

[5] L.A. Klein. Data and sensor fusion: A tool for information assessment and decision making. Bellingham, Wash: SPIE Press.(2004)

[6] - Franklin E. White, Jr., Data Fusion Lexicon, Joint Directors of Laboratories, Technical Panel for C3, Data Fusion Sub-Panel, Naval Ocean Systems Center, San Diego, 1987.

[7] Erik P. Blasch, Eloi Bosse, and Dale A. Lambert (2012), High-Level Information Fusion Management and System Design, Artech House Publishers, Norwood, MA. ISBN 1608071510 | ISBN 9781608071517

[8] H. B. Mitchell (2007) Multi-sensor Data Fusion - An Introduction, Springer-Verlag, Berlin, ISBN 978-3-540-71463-7

[9] B. Dasarathy, Decision Fusion,New York: IEEE Computer Society Press, 1994.

[10] A. Steinberg and C. Bowman, "Revisions to the JDL data fusion processing model,' in Handbook of Multisensor Data Fusion,D.Hall and J. Llinas, Eds., Boca Raton, FL: CRCPress, 2001, Chapter 2.

[11] Wilfried, E. 2002. An introduction to sensor fusion. Research report 47/2001 . Austria: Institute fur Technische Informatik, Vienna University of Technology.

[12] Markin, M., C. Harris, M. Bernhardt, J. Austin, M. Bedworth, P. Greenway, R. Johnston, et al. 1997. Technology foresight on data fusion and data processing. Publication of The Royal Aeronautical Society.

[13] Boyd, R. John, ADiscourse on Winning and Losing,August 1987, Private Collections. Master copyin LibraryatMarine Corps University, Quantico, VA.

[14] G. Hammond, The Mind of War: John Boyd and American Security,Washington, DC: Smithsonian Institution Press, May2001.

[15] Thomopoulos, S. C. Sensor integration and data fusion. Proc. SPIE 1198, Sensor Fusion II: Human and Machine Strategies, 1989: 178191.

[16] Multi Sensor Data Fusion - Hugh Durrant-Whyte - Australian Centre for Field Robotics The University of Sydney NSW 2006 Australia hugh@acfr.usyd.edu.au January 22, 2001.

[17] Bedworth, M. and O'Brien, J, 1999, The Omnibus model: a new model of data fusion? Proc. 2nd Intl. Conf. on Information Fusion, Sunnyvale

[18] Endsley, M.R. (1995b). Toward a theory of situation awareness in dynamic systems. Human Factors 37(1), 32-64.

[19] Artman, H. (2000). Team situation assessment and information distribution. Ergonomics, 43(8), 1111-1128.

[20] Multi-sensors data fusion using Dynamic Bayesian Network for robotised vehicle geo-localisation Cappelle, C. ; El Najjar, M.E. ; Pomorski, D. ; Charpillet, F. Information Fusion, 2008 11th International Conference on Publication Year: 2008, Page(s): 1 - 8

[21] Sensor planning for mobile robot localization using Bayesian network representation and inference Hongjun Zhou; Sakane, S. Intelligent Robots and Systems, 2002. IEEE/RSJ International Conference on Volume: 1 DOI: 10.1109/IRDS.2002.1041430 Publication Year: 2002 , Page(s): 440 - 446 vol.1

[22] Multi-sensor Fusion Method Using Bayesian Network for Precise Multi-vehicle Localization Smaili, C. ; El Najjar, M.E. ; Francois Intelligent Transportation Systems, 2008. ITSC 2008. 11th International IEEE Conference on DOI: 10.1109/ITSC.2008.4732643 Publication Year: 2008 , Page(s): 906 911
[23] Multi-sensor Fusion Method Using Dynamic Bayesian Network for Precise Vehicle Localization and Road Matching Smaili, C. ; El Najjar, M.E. ; Charpillet, F. Tools with Artificial Intelligence, 2007. ICTAI 2007. 19th IEEE International Conference on Volume: 1 DOI: 10.1109/ICTAI.2007.70 Publication Year: 2007 , Page(s): $146-151$

[24] Bayesian Train Localization Method Extended By 3D Geometric Railway Track Observations From Inertial Sensors Oliver Heirich, Patrick Robertson, Adrian ' Cardalda Garc' .a, Thomas Strang DLR (German Aerospace Center) Institute of Communications and Navigation 82234 Wessling, Germany

[25] An Effective Kalman Filter Localization Method for Mobile Robots SangJoo Kwon ; Kwang Woong Yang; Sangdeok Park Intelligent Robots and Systems, 2006 IEEE/RSJ International Conference on DOI: 10.1109/IROS.2006.281982 Publication Year: 2006 , Page(s): $1524-1529$

[26] Extended Kalman filter based pedestrian localization for collision avoidance Xu, Y.W. ; Cao, X.B. ; Li, T. Mechatronics and Automation, 2009. ICMA 2009. International Conference on DOI: 10.1109/ICMA.2009.5246613 Publication Year: 2009 , Page(s): $4366-4370$

[27] GPS aided Extended Kalman Filter based localization for unmanned vehicles Tuna, G. ; Gungor, V.C. ; Gulez, K. Signal Processing and Communications Applicatons Conference (SIU), 2012 20th DOI: 10.1109/SIU.2012.6204515 Publication Year: 2012 , Page(s): 1 - 4

[28] RailSLAM - Localization of rail vehicles and mapping of geometric railway tracks Heirich, O. ; Robertson, P. ; Strang, T. Robotics and Automation (ICRA), 2013 IEEE International Conference on DOI: 10.1109/ICRA.2013.6631322 Publication Year: 2013 , Page(s): 5212 - 5219

[29] Unscented information filter based multi-sensor data fusion using stereo camera, laser range finder and GPS receiver for vehicle localization Lijun Wei ; Cappelle, C. ; Ruichek, Y. Intelligent Transportation Systems (ITSC), 2011 14th International IEEE Conference on DOI: 10.1109/ITSC.2011.6082990 Publication Year: 2011 , Page(s): 1923 - 1928

[30] An Interlaced Extended Information Filter for Self-Localization in Sensor Networks Gasparri, A. ; Pascucci, F. Mobile Computing, IEEE Transactions on Volume: 9 , Issue: $10 \quad$ DOI: 10.1109/TMC.2010.122 Publication Year: 2010 , Page(s): 1491 1504

[31] Global pose localization of an autonomous wheeled mobile robot by fusing active RFID and ranging laser scanner: A fuzzy extended information filtering approach Hung-Hsing Lin ; Ching-Chih Tsai ; Yi-Yu Li SICE Annual Conference, 2008 DOI: 10.1109/SICE.2008.4655169 Publication Year: 2008, Page(s): 2956 $-2961$

[32] Mobile robot localization using fuzzy neural network based extended Kalman filter Thi Thanh Van Nguyen ; Manh Duong Phung ; Thuan Hoang Tran ; Quang Vinh Tran Control System, Computing and Engineering (ICCSCE), 2012 IEEE International Conference on DOI: 10.1109/ICCSCE.2012.6487181 Publication Year: 2012 , Page(s): 416 - 421

[33] Multi-Sensor Fuzzy Data Fusion Using Sensors with Different Characteristics Akhoundi, Mohammad Amin Ahmad; Valavi, Ehsan eprint arXiv:1010.6096 10/2010 ARXIV

[34] Multi-Sensor Data Fusion in Cluster based Wireless Sensor Networks Using Fuzzy Logic Method Manjunatha, P. ; Verma, A.K. ; Srividya, A. Industrial and Information Systems, 2008. ICIIS 2008. IEEE Region 10 and the Third international Conference on DOI: 10.1109/ICIINFS.2008.4798453 Publication Year: 2008 , Page(s): 1 - 6

[35] Multi-sensor data fusion for helicopter guidance using neuro-fuzzy estimation algorithms Doyle, R.S. ; Harris, C.J. Systems, Man and Cybernetics, 1995. Intelligent Systems for the 21st Century., IEEE International Conference on Volume: 2 DOI: 10.1109/ICSMC.1995.537967 Publication Year: 1995 , Page(s): $1392-1397 \mathrm{vol} .2$ 
[36] Based on fuzzy neural network of multi-agent data fusion Ma, Bin ; Li, Nannan; Wang, Changtao ; Han, Zhonghua ; Tongying Guo Modelling, Identification \& Control (ICMIC), 2012 Proceedings of International Conference on Publication Year: 2012 , Page(s): 975 980
[37] FuTRO Challenges- Future Railway. Available at: www.futurerailway.org/.../FuTRO\%20Innovation $\% 20$ Competition $\% 2$ 0A... (Accessed: 14/01/2014/). 
2015

\section{Data fusion strategy for precise vehicle location for intelligent self-aware maintenance systems}

\section{Bevilacqua, Maurizio}

Institute of Electrical and Electronics Engineers

Bevilacqua $\mathrm{M}$ et al. Data fusion strategy for precise vehicle location for intelligent self-aware maintenance systems, Proceedings of the 2015 6th International Conference on Intelligent Systems, Modelling and Simulation. 9 - 12 February 2015, Kuala Lumpur, Malaysia.

https://dspace.lib.cranfield.ac.uk/handle/1826/10669

Downloaded from Cranfield Library Services E-Repository 